\title{
Dependence on working pressure of amorphous GaAs prepared by RF magnetron sputtering
}

\author{
Yanping Yao \\ College of Information and Technology \\ Jilin Normal University, \\ Siping, china \\ e-mail: meyanping@163.com
}

\author{
Baoxue Bo \\ State Key Lab on High Power Semiconductor Lasers \\ Changchun University of Science and Technology \\ Changchun, china \\ e-mail: bobaoxue@yahoo.com
}

\begin{abstract}
We prepared amorphous GaAs ( $a$-GaAs) films by an RF magnetron sputtering method and investigated the influence of working pressure on the structure, composition, optical and electrical properties. The experimental data suggest the films prepared above $0.5 \mathrm{~Pa}$ are amorphous and the Ga to As concentration ratio decreases when increasing working pressure .The optical gap, resistivity and photosensitive increase with increasing working pressure. These findings suggest that working gas pressure is very important to control structure, chemical composition, optical and electrical properties of $a$-GaAs films prepared by magnetron sputtering method. These results were analyzed in terms of the effect of gas pressure on defect and band-tail state densities in $a$-GaAs films.
\end{abstract}

Keywords-Amorphous;GaAs;properties; working pressure ; structure

\section{INTRODUCTION}

The improvement of the electronic properties of amorphous $\mathrm{GaAs}(a-\mathrm{GaAs})$ films in electronic devices has attracted considerable interest. Thus far, this material has been used as an antiguide layers in vertical cavity surfaceemitting lasers[1], as a buffer layer between silicon and gallium arsenide epitaxial layers[2,3], and as a host material for $\mathrm{Er}$ doping[4]. A material appropriate to electronic applications does not have a high density of defects. In amorphous semiconductors defects are generally compensated by hydrogen incorporation or by high-temperature deposition[5-10]. At present, the reduction of deep defect and shallow band-tail state densities is one of the major research goals in amorphous semiconductors with improved transport properties.

The work presented in this article extends the earlier work on amorphous III-V semiconductors. In a previous article, we reported that the effects of hydrogenation on $a$ GaAs[11]. In this article, we study the effect of working pressure on the structure, composition, optical and electrical properties of $a$-GaAs. The deposition system used and the details of sample preparation and measurement procedures have been described in this article. We intend to yielded moderate improvements in optical and electrical properties of $a$-GaAs film through optimizing working pressure. The effect of working pressure on defect and band-tail state densities in $a$-GaAs films is discussed.

\section{EXPERIMENTAL DETAILS}

Amorphous GaAs films were deposited in a RF magnetron sputtering system in Ar ambient. A pure GaAs $(99.999 \%)$ wafer with diameter of $50 \mathrm{~mm}$ was used as the sputtering target. Quartz glass was selected as substrate. These films were deposited at room temperature. Film growth was carried out in the growth ambient with working pressure ranging from 0.25 to $8 \mathrm{~Pa}$ and a RF power of $100 \mathrm{~W}$. The distance between the target and substrate was fixed at $4 \mathrm{~cm}$. Deposition time was $2 \mathrm{~h}$.

Structural properties of thin film were studied by D/MAX-IIB X-ray diffractometer (XRD) using $\mathrm{Cu} \mathrm{Ka}$ radiation over a range of $2 \theta=10-80^{\circ}$. Their chemical composition was determined by a scanning electron microscope (JSM-840) equipped with Link ISIS-30 an energy-dispersive X-ray spectrometer (EDX). The optical transmittance and reflectance spectra were measured by a double beam spectrophotometer (UV2150PC) over spectral range of $210-3000 \mathrm{~nm}$. Resistivity and carrier concentration were checked by Hall system. Photosensitivity properties were carried out on films with evaporated gold electrodes, using a measurement system including a microampere meter, an illumination lamp and a sample chamber.

\section{RESULTS AND DISCUSSION}

\section{A. Structure and composition}

We have studied the effect of the working pressure on the crystallographic structure of the deposited films, as shown in Fig.1. For films prepared at $0.25 \mathrm{~Pa}$, XRD peaks of ( $\left.\begin{array}{lll}1 & 1 & 1\end{array}\right)$ was observed. On the other hand, no XRD peak was observed for films prepared above $0.5 \mathrm{~Pa}$. These findings reveal that films prepared at $0.25 \mathrm{~Pa}$ were microcrystalline, while films prepared above $0.5 \mathrm{~Pa}$ were amorphous.

We have studied the effect of $P_{w}$ on the composition of the deposited films. The $\mathrm{Ga}$ to As concentration ratio versus $P_{w}$ is shown in Fig.2. We can see that the Ga to As concentration ratio decreases when increasing $P_{w}$. During the transport process of the sputtered atoms, one of the two components may be more rapidly thermalized by collisions with argon atoms[12], leading to a sputtered film having a deficiency of the more thermalized component. For $\mathrm{Ga}$ and As atom, the energy loss by collisions with argon atoms at a distance $z$, from the target, 
can be written as

$$
\begin{gathered}
E_{G a}\left(E_{0}, z, \theta\right)=E_{0}\left(1-\frac{z P_{w}}{0.467 T \sqrt{E_{0}} \cos \theta}\right)^{2} . \\
E_{A s}\left(E_{0}, z, \theta\right)=E_{0}\left(1-\frac{z P_{w}}{0.47 T \sqrt{E_{0}} \cos \theta}\right)^{2} .
\end{gathered}
$$

Where $E_{0}$ is the initial energy of the atom, $\theta$ its angular direction and $T$ electronic temperature of the plasma.The electronic temperature of the plasma is around 8ev We find that the same reduction of the energy of As requires a higher $z P_{w}$ than that in the case of Ga. This result shows that, in the same experimental conditions, Ga atoms are thermalized on a smaller distance than As atoms. The sputtered $\mathrm{Ga}$ atoms have a higher probability of being thermalized than that of the sputtered As atoms when $P_{w}$ increases. So, Ga to As concentration ratio decreases with $P_{w}$ increasing.

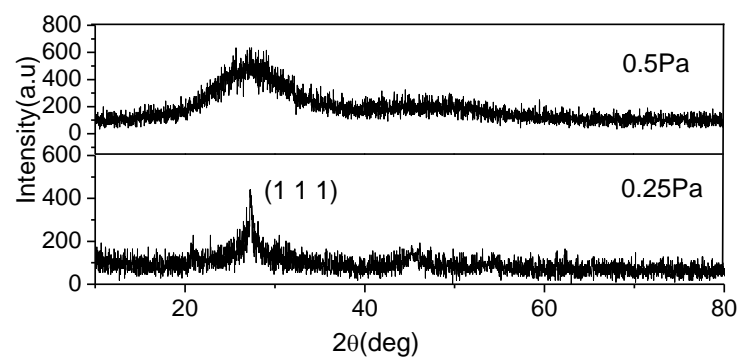

Figure 1. XRD spectra of GaAs thin films.

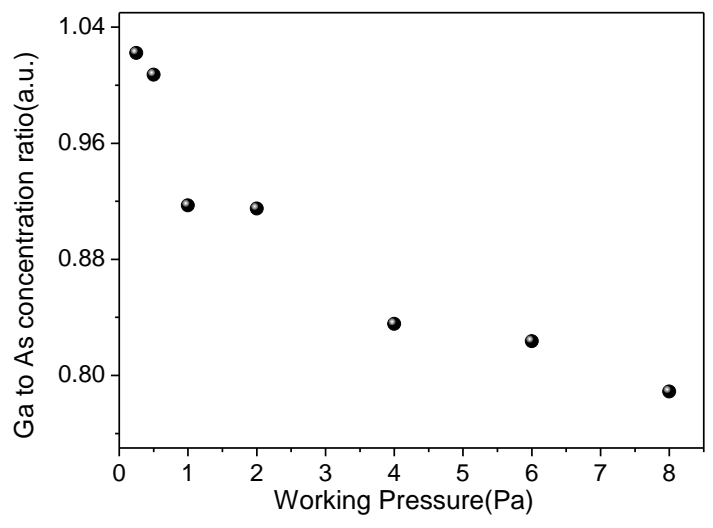

Figure 2. The Ga to As concentration ratio versus $P_{w}$.

\section{B. The optical gap of a-GaAs}

The measurement of transmission spectra and reflection spectra are carried out by spectrophotometer at 210-3000 $\mathrm{nm}$. So we have obtain transmittance $T$ and reflectance $R$.
Fig.3 show the transmission spectra of GaAs thin films prepared at $0.25-5 \mathrm{~Pa}$.

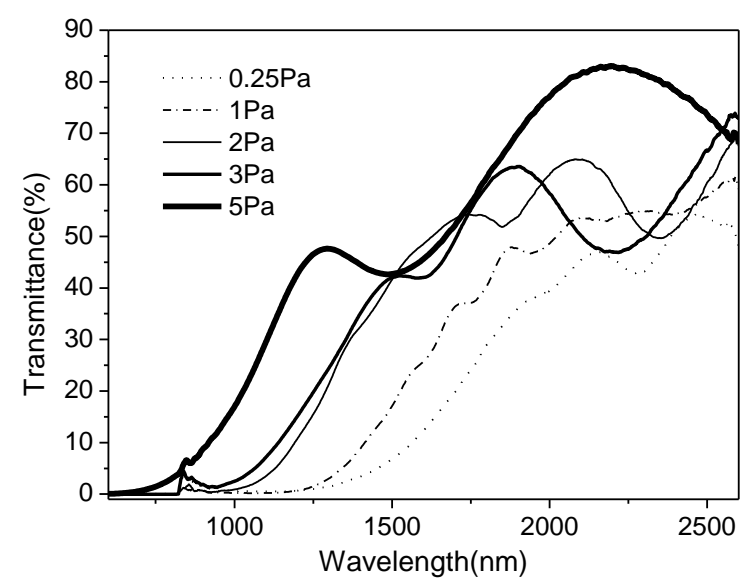

Figure 3. The transmission spectra of GaAs thin films

The absorption coefficient $\alpha$ are given by

$$
\alpha=\frac{-\ln \left[T /(1-R)^{2}\right]}{d} .
$$

Where $d$ is thickness of film examined by profilometer. For the determination of the optical gap, we employ the "Tauc" approach[13]. That is, we fit, in a least-squares sense

$$
(\alpha(h v) h v)^{1 / 2}=B\left(h v-E_{o p t}\right)
$$

to the relevant experimental data, where $B$ denotes the slope of the Tauc extrapolation and $E_{\text {opt }}$ represents the corresponding Tauc optical gap. The $h v$ is photon energy. $B$ is constant and is inversely proportional to its conduction band tail and valence band tail. The value of $B$ is generally between $10^{5} \mathrm{~cm}^{-1} \mathrm{eV}^{-1}$ and $10^{6} \mathrm{~cm}^{-1} \mathrm{eV}^{-1}$. The quantitative evaluation of $B$ and $E_{o p t}$ depend critically upon the range of data over which the fit of (4) is taken. For the purposes of this analysis, we perform our fit of (4) to the experimental data for which $10^{3} \mathrm{~cm}^{-1} \leq \alpha(h v) \leq 10^{4} \mathrm{~cm}^{-1}$. In the this absorption region, the relationship curve basically is a straight line. The linear extrapolation to the shaft, the intercept is the optical optical gap, as shown in Fig.4. Fig.4(a) and (b) are the Tauc fits corresponding to $a$-GaAs samples prepared at $0.5 \mathrm{~Pa}$ and $2 \mathrm{~Pa}$.

The optical gap and $B$ values derived from fits of $(\alpha(h v) h v)^{1 / 2}-h v$ are shown in Fig.5. $E_{\text {opt }}$ is between 1.237 and $1.4 \mathrm{eV}$. The optical gap of GaAs is affected by two factors: the composition and local bond ordering. We have studied the effect of the working pressure $P_{w}$ on the composition, as shown in Fig.2. 

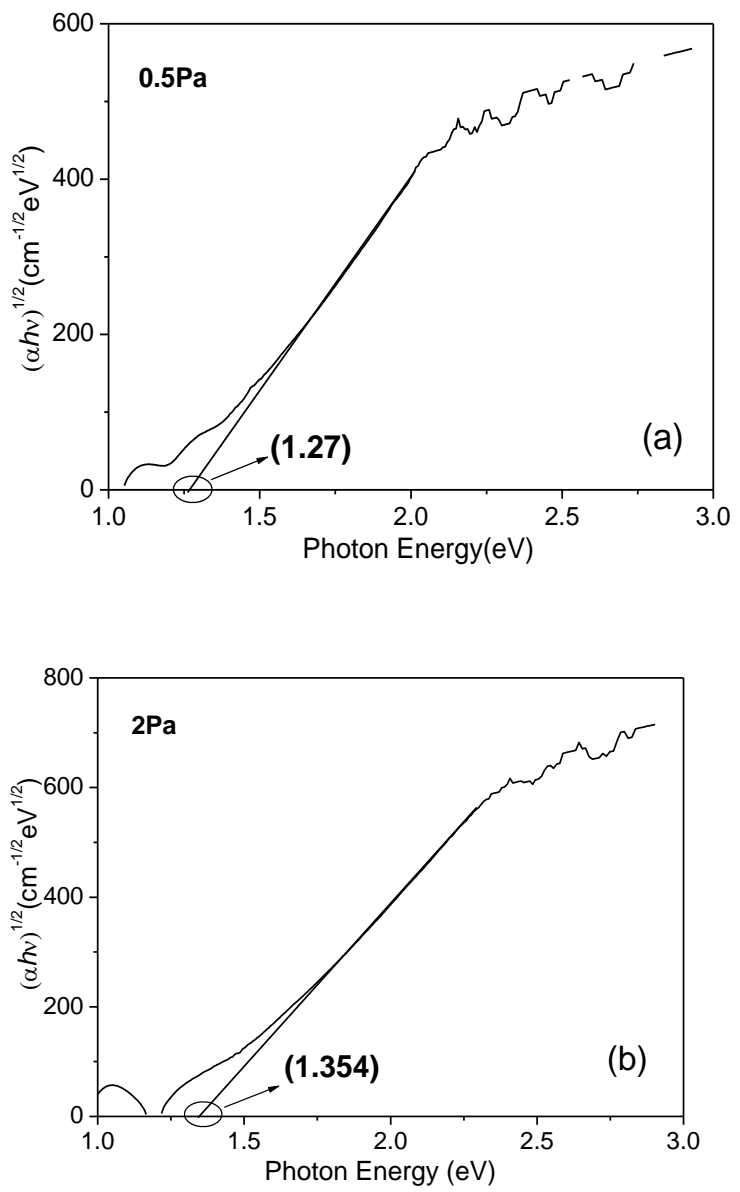

Figure 4. The Tauc fits of $a$-GaAs samples prepared at (a) $0.5 \mathrm{~Pa}$ and (b) $2 \mathrm{~Pa}$.

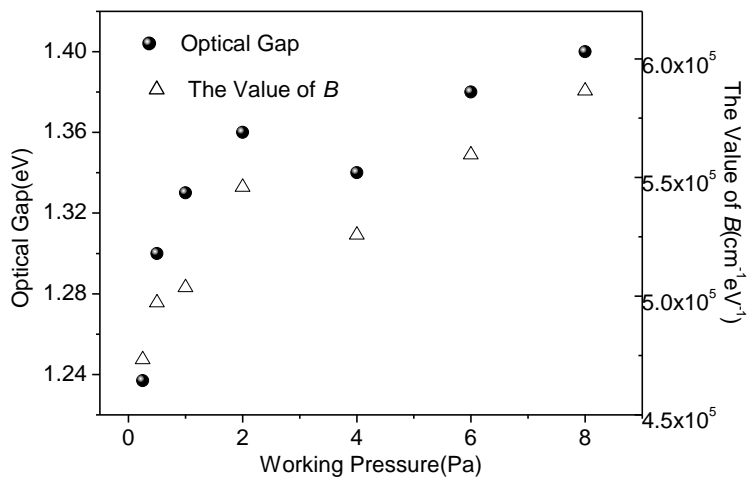

Figure 5. The effect work pressure on the optical optical gap and B.

The films contain an excess of As when $P_{w}$ is higher than $1 \mathrm{~Pa}$. And excess of As increases with the increase of $P_{w}$. But optical gap value show little variation with composition. Baker[14,15] interpret this as defect states related rich As lying in energy band or deep band tail. So rich As has no great influence on the absorption edge. As the working pressure increases, the optical gap increases. The increase of $P_{w}$ increase the collision with Ar atom and the energy of the sputtered atoms decreases as reaching the substrate. The results are consistent with the conclusion of Zaka et al [16]. Zaka et al have pointed out that the optical optical gap increases with the pressure increasing. Zaka et al explain the effect of $P_{w}$ on the optical optical gap of the films, from the substrate surface dynamics. As $P_{w}$ increases, the energy of various particle decreases which reduces the number of defects center. Other researchers have studied effect of working pressure on the properties of the magnetron sputtering $a$-Si film and obtained the same conclusion. We characterize the band tails using the slope of the exponential region of the optical absorption coefficient, $B$. Information about the band tail can be inferred from the quantity $B$. This $\Delta E$ is primarily determined by optical transitions involving the band tails, and closely matches the slope of the wider of the two band tails. $\Delta E$ provides an estimate of the total density of band tails states. And we have calculated $B$ $\left(3.46 \times 10^{5}-4.18 \times 10^{5} \mathrm{~cm}^{-1} \mathrm{eV}^{-1}\right)$, as shown in Fig.5.

With $B$ increasing, $\Delta E$ reduces, indicating defects states density of band tails decreases. The $B$ value is compared to an set of amorphous materials, as shown inTable I.

TABLE I. THE $B$ TYPICAL VALUE OF AMORPHOUS MATERIALS

\begin{tabular}{|c|c|c|c|c|c|}
\hline & $\boldsymbol{a}$-GaAs & $\boldsymbol{a}$-Si & $\boldsymbol{a}$-Si:H & $\boldsymbol{a}$-As $\boldsymbol{S}_{\mathbf{3}}$ & $\boldsymbol{a}$ - $\boldsymbol{A s}_{2} \mathbf{S e}_{\mathbf{3}}$ \\
\hline $\begin{array}{c}E_{\text {opt }} \\
(\mathrm{eV})\end{array}$ & 1.3 & 1.26 & 1.82 & 2.32 & 1.76 \\
\hline $\begin{array}{c}\mathrm{B} \\
\left(\times 10^{5} \mathrm{~cm}^{-1} \mathrm{eV}^{-1}\right)\end{array}$ & 4.97 & 5.3 & 4.6 & 4 & 8.3 \\
\hline
\end{tabular}

\section{The electrical properties}

We investigated the influence of working pressure on the room temperature resistivity and carrier concentration, as shown in Fig.6.With the increasing of working pressure, resistance increases while the carrier concentration decreased because the disordering degree of structure decreases.

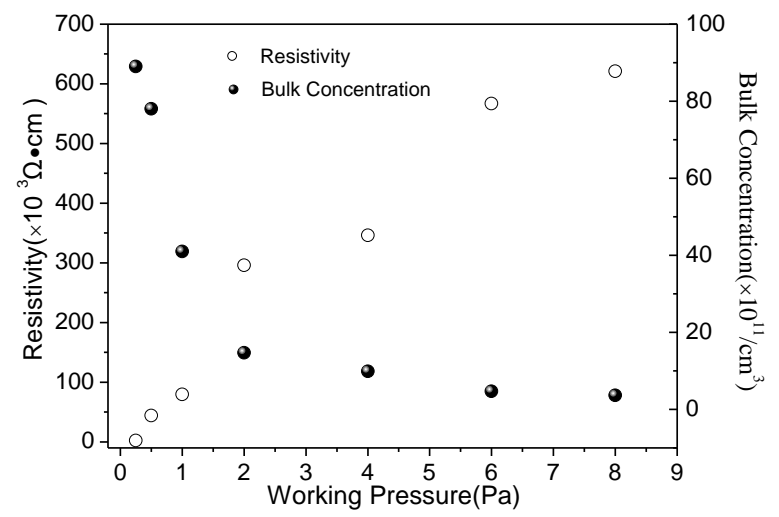

Figure 6. The influence of working pressure on the resistivity and carrier concentration 


\section{Photosensitivity}

The value of photosensitivity for a photoelectric material determines the application of the photoconductive devices. Dark current, $i_{d}$, was the current of thin films under no light lumination condition. Photocurrent, $i_{p}$, was the additional current of the excess number of photogenerated charge carriers under steadystate illumination, which was gained by subtracting dark current from the current of lumination. The photosensitivity, $\eta_{p}$, is defined as

$$
\eta_{p}=i_{p} / i_{d}
$$

The dependence of photoconductivity with working pressure in $a$-GaAs thin film is shown in Fig.7.

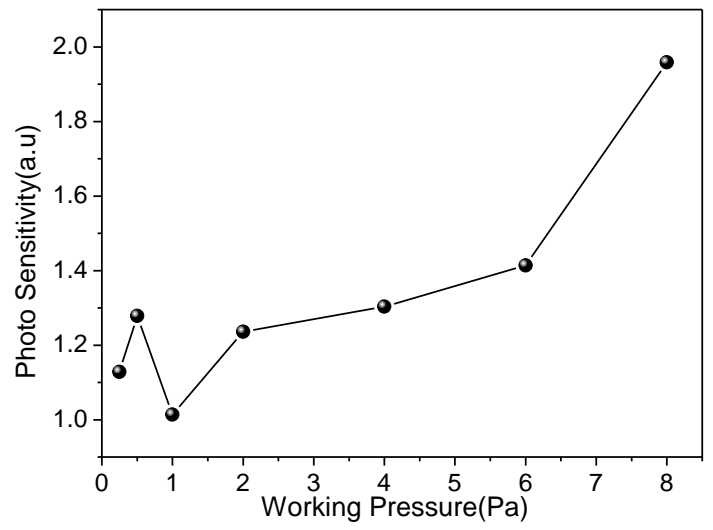

Figure 7. The influence of working pressure on the resistivity and carrier concentration.

The photosensitive in the $a$-GaAs film is from 1.5 to 2 . $a$-GaAs film has obvious photosensitive and the photosensitive with the pressure increasing. The recombination centers have a significant influence on photosensitive properties. The recombination centers have two component, structural defects and component defects. We analyzed the film component, shown in Fig.2. The film composition close to stoichiometric at $0.5 \mathrm{~Pa}$, the photosensitivity is highest in the low pressure range (less than 4Pa). The increasing of $P_{w}$ increase the collision with Ar atom and the initial energy of the sputtered atoms decreases, thus the structural disorder degree increases and structural defects decreases. The pressure $P_{w}$ is larger than $1 \mathrm{~Pa}$, the photosensitive properties increased with the increasing of pressure. As rich films increases significantly at the $1 \mathrm{~Pa}$, while the structural defects did not significantly reduced with the higher energy of the sputtered atoms. So the photosensitive is not obvious. The increasing of $P_{w}$ can minimize the energy of the sputtered particles and can effectively improve the photosensitive.

\section{CONCLUSION}

We prepared $a$-GaAs films by an RF magnetron sputtering method and investigated the influence of gas pressure on the structural, composition, optical properties and electrical properties during the deposition. The X-ray diffraction reveal that films prepared at $0.25 \mathrm{~Pa}$ were microcrystalline and films prepared above $0.5 \mathrm{~Pa}$ were amorphous. The EDX analysis showed Ga to As concentration ratio decreases with $P_{w}$ increasing. As the working pressure increases, the optical gap, resistance and photosensitive increases. The effect of $P_{w}$ is explained from the substrate surface dynamics. As $P_{w}$ increases, the energy of various particle decreases which reduces the number of defects center. These investigations suggest that gas pressure is very important to control optical properties and electrical properties of $a$-GaAs films prepared by magnetron sputtering method.

\section{REFERENCES}

[1] Choo K I, Choo W K, Lee J Y, Park S C, and Nishinaga T, "Defect formation in the solid phase epitaxial growth of GaAs films on $\mathrm{Si}$ (001) substrate ," Journal of applied physics, vol.69(1), pp. 237242, 1991.

[2] Aguir K, Fennouh A, Carchano H, Seguin JL, El Hadadi B and Lalande F, "Electrical properties of a-GaAs/c-Si (p) heterojunctions," Thin Solid Films , vol. 257(1), pp. 98-103, 1995.

[3] Fennouh A, Aguir K, Carchano H and Sguin JL, "Electrical characteristics of amorphous GaAs-n-crystalline S heterojunctions," Materials Science and Engineering: B, vol. 24(1), pp. 27-31, 1995

[4] Yoo B S, Chu HY, Park H H, Lee H G and Lee, "Transverse mode characteristics of vertical-cavity surface-emitting lasers buried in amorphous GaAs antiguide layer ," Quantum Electronics, IEEE Journal of, vol. 33(10), pp. 1794-1800, 1997.

[5] Benachir El Hadadi, Herve Carchano, Jean-Luc Seguin and Hassan Tijani, "Structural and electrical properties of amorphous GaAs sputtered at high substrate temperature," Vacuum, vol. 80, pp 272-283, 2005

[6] S. J. Pearton, J. W. Corbett and T. S. Shi, "Hydrogen in crystalline semiconductors ,'Applied Physics A, vol. 43(3), pp. 153-195, July , 1987.

[7] H. Y. Cho,E. K. Kim, Y. Kim, H. S. Kim, M. S. Kim, and S. K. Min, "Metastable behavior of deep levels in hydrogenated GaAs," Applied physics letters, vol. 58(17), pp. 1866-1868, Jan., 1991.

[8] Bube R H and Bube R H, Photovoltaic materials. Amsterdam: Imperial College Press, 1998.

[9] Street, Robert A, Hydrogenated amorphous silicon. Cambridge University Press, 2005.

[10] Capezzuto, Pio, Arun Madan, Plasma deposition of amorphous silicon-based materials. Academic Press, 1995.

[11] YAO Yan-Ping, LIU Chun-Ling,QIAO Zhong-Liang, LI Mei,GAO Xin and BO Bao-Xue, "Structural, Optical and Electrical Properties of Hydrogen-Doped Amorphous GaAs Thin Films,' Chinese Physics Letters. vol. 3 (25), pp. 1071-1074, March, 2008.

[12] Seguin, J. L., Hadadi, B. E., Carchano, H., and Aguir, K, "Composition study of high temperature sputtered amorphous $\mathrm{Ga}_{\mathrm{x}} \mathrm{As}_{1-\mathrm{x}}$ films," Journal of non-crystalline solids, vol. 238(3), pp. 253-258, September, 1998.

[13] Tauc J, Grigorovici R, and Vancu A, "Optical properties and electronic structure of amorphous germanium,” Phys. Status Solidi (b), vol. 15(2), pp. 627-637, March, 1966.

[14] S.H. Baker, M.I. Manssor, and S.J. Gurman, "Structural investigation of the $a-\mathrm{Ga}_{1-x} \mathrm{As}_{x}$ system," J. Non-Cryst. Solids, vol. 144, pp. 63-69, 1992.

[15] Baker S H, Spear W E, Gibson R A G, "Electronic and optical properties of a-Si1-xCx films prepared from a H2-diluted mixture of SiH4 and CH4," Philosophical Magazine B, vol. 62(2) , pp.213223. 1990

[16] Y. Zaka, S.A.Abo-Namous, and R.W.Fane, "Control of electrical and optical properties of unhydrogenated neon-sputtered amorphous silicon by preparation conditions," Thin Solid Films, vol. 125(1-2), pp. 47-52, March, 1985 